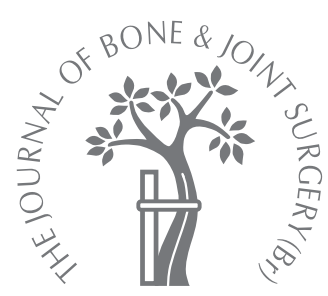

\title{
Urinary catheter tamponade to control intra- operative bleeding from the superior gluteal
} artery

\author{
A CASE REPORT
}

S. Konangamparam -bath, J. M. Wilkinson, T. Cleveland, I. Stockley

From Northern General Hospital, Sheffield, England

In. Konangamparambath, MBBS, Senior House Officer I. M. Wilkinson, PhD, FRCS(Tr \& Orth), Hip Fellow - I. Stockley, MD, FRCS, Consultant Orthopaedic Surgeon

Department of Orthopaedics T. Cleveland, FRCS, FRCR, Consultant Interventional Radiologist

Sheffield Vascular Institute Northern General Hospital, Herries Road, Sheffield S5 7AU, UK.

Correspondence should be sent to $\mathrm{Mr} \mathrm{J}$. M. Wilkinson; e-mail: wilkomark@aol.com

()2007 British Editorial Society of Bone and Joint Surgery doi:10.1302/0301-620X.89B1. $18162 \$ 2.00$

$J$ Bone Joint Surg [Br] 2007;89-B: 32-3.

Received 23 May 2006;

Accepted 23 August 2006

\author{
Bleeding is a major complication of revision total hip replacement. We report a case where \\ the inflated balloon of a urinary catheter was used to temporarily control intrapelvic \\ bleeding from the superior gluteal artery, while definitive measures for endovascular \\ embolisation were made.
}

Revision total hip replacement (THR) carries significant risks, including infection, bleeding, nerve injury and thromboembolism. Dealing with intra-operative bleeding during revision surgery can be challenging, particularly when the source is intrapelvic and cannot be seen or controlled. Methods described for the control of bleeding include ligation of the artery, diathermy, and percutaneous transcatheter embolisation of the artery. Effective control by embolisation requires temporary control in order to allow the patient to be transferred to an angiography suite from the operating theatre. Here, we describe a novel method of controlling intra-operative bleeding from the superior gluteal artery by using a Foley catheter (CR Bard Inc., Murray Hill, New Jersey) to tamponade the vessel against the inner table of the greater sciatic notch.

\section{Case report}

A 69-year-old hypertensive woman underwent revision of a failed acetabular reconstruction because of pelvic dissociation. It was her fourth revision, the index replacement having taken place in 1989. In 2001 she underwent exchange of the acetabular component, with impacted morcellised allograft bone and mesh, in order to reconstruct the acetabulum. There was significant bone lysis involving both bony columns. A further two revisions followed in 2002 after mechanical failure of the reconstruction.

The procedure was carried out under general anaesthesia with the patient in the lateral position. A posterior approach was used to expose the hip. On removal of the acetabular component, an unstable dissociation passing through the greater sciatic notch, and crossing the acetabulum transversely, was identified.

The superior gluteal artery was accidentally injured while stripping scarred soft tissues from the posterior column of the acetabulum at the greater sciatic notch, in preparation for applying a posterior-column reconstruction plate. This resulted in uncontrolled haemorrhage and cardiovascular instability. Intrapelvic retraction of the cut vessel made it difficult to identify the bleeding point. Digital pressure against the inner table of the greater sciatic notch was used to control the bleeding temporarily. A size 14 Fr Foley catheter was inserted into the greater sciatic notch and the balloon inflated with $10 \mathrm{ml}$ of saline. This achieved tamponade of the bleeding vessel against the bone and the patient became haemodynamically stable. Gauze swabs were packed into the wound, which was then partially closed and the patient transferred to the angiography suite.

Initial angiography, from a left femoral approach, failed to demonstrate bleeding, because of the successful tamponade effected by the balloon of the Foley catheter (Fig. 1). There was concern that deflation of the balloon might cause loss of control of the bleeding, so an occlusion balloon $(7 \mathrm{~mm})$ was passed down the internal iliac artery, using the same left femoral approach. After deflation of the Foley balloon, rapid extravasation of contrast was identified (Fig. 2). The Foley balloon was reinflated and the superior gluteal branches selectively embolised using coils (3 $\mathrm{mm}$ and $5 \mathrm{~mm}$ diameters, respectively; Cook Inc., Bloomington, New Jersey). Successful embolisation was confirmed with both the Foley and internal iliac balloons deflated (Fig. 3).

The patient was returned to the operating theatre later that day and the hip reconstruction was completed. Subsequent post-operative recovery was unremarkable and she was discharged six days later. 


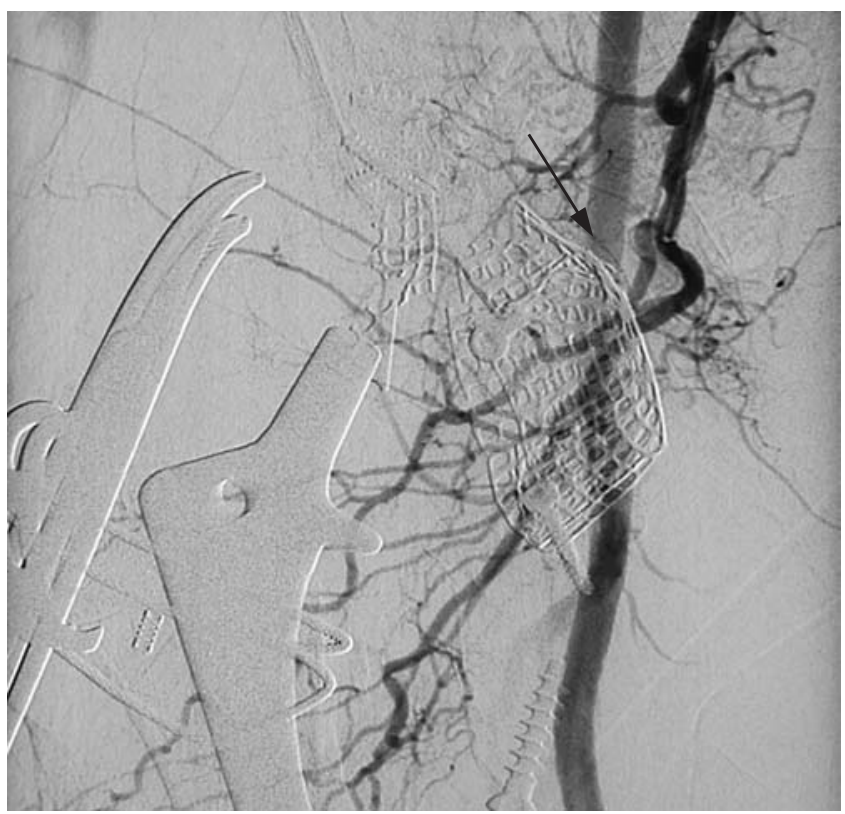

Fig. 1

Contrast angiogram showing no evidence of bleeding because of successful tamponade of the superior gluteal artery by the Foley catheter against the inner table of the greater sciatic notch. The artery forceps used to apply traction to the catheter is shown, as well as the acetabular mesh and femoral component.

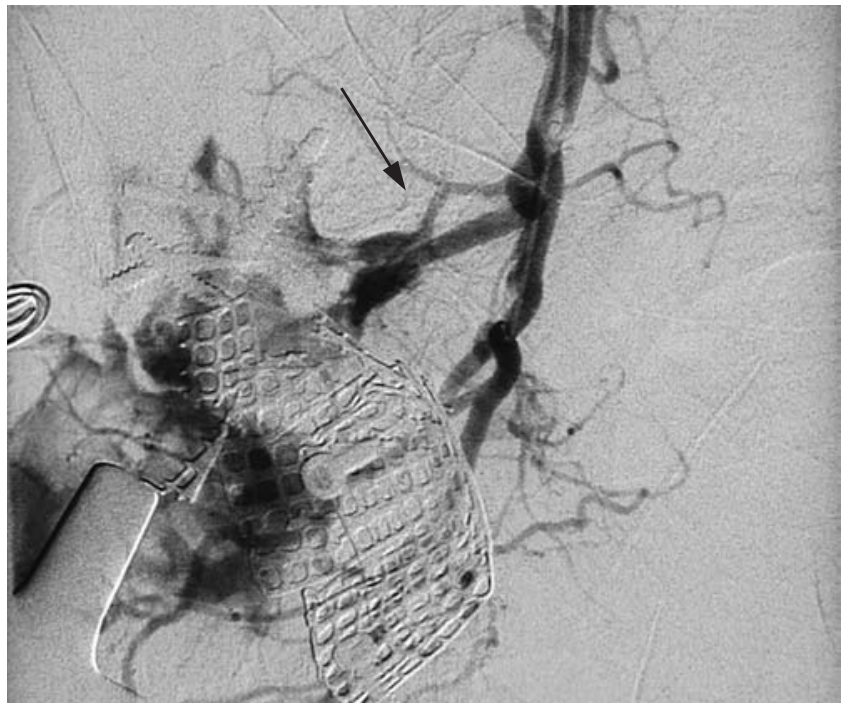

Fig. 2

Contrast angiogram with the balloon of the Foley catheter deflated, confirming the superior gluteal artery as the site of the bleeding.

\section{Discussion}

The superior gluteal artery is the largest branch of the internal iliac artery. It leaves the pelvis above the piriformis muscle, through the great sciatic foramen, which is formed by the greater sciaticnotch anteriorly, the sacrotuberous ligament posteriorly, and the sacrospinous ligament and sacral

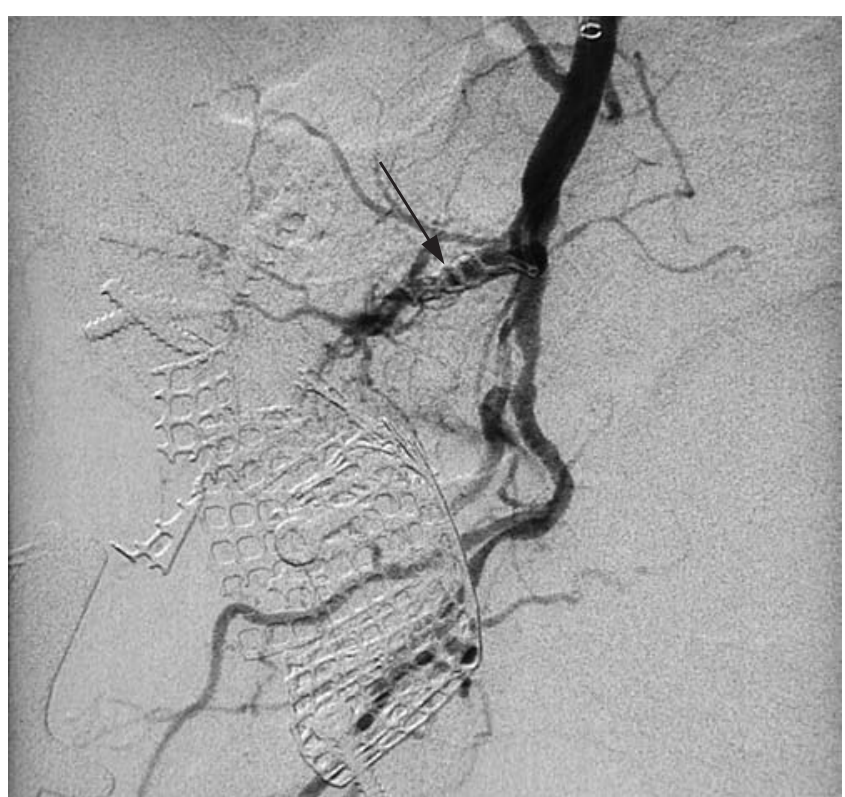

Fig. 3

Contrast angiogram confirming haemostasis by coil embolisation of the bleeding vessels. Both the Foley and internal iliac balloons have been deflated.

spine inferiorly. The vessel's close proximity to the acetabulum, its angulation as it passes out of the pelvis, and the small quantity of muscle that protects it are said to increase its rise of injury in pelvic trauma. ${ }^{1}$ The same situation exists at revision THR. Patients with atherosclerosis and those who have previously undergone orthopaedic procedures or who have suffered injuries have a higher risk of iatrogenic arterial injuries. ${ }^{2}$ Once bleeding occurs, digital pressure should be used to stop it and the ends of the vessel should then be identified. Ligature or diathermy may be used. Should it not be possible to identify the bleeding site, endovascular angiography can be useful to identify the source and control the bleeding by embolisation. ${ }^{3,4}$ However, it is essential that the patient is haemodynamically stable before they leave the operating theatre. The method we describe here has not previously been reported, although there are clearly many unpublished examples of the use of pressure to control bleeding. We believe our use of a Foley catheter is a simple technique that can be used to control bleeding while assistance is sought.

No benefits in any form have been received or will be received from a commercial party related directly or indirectly to the subject of this article.

\section{References}

1. Miller WE. Massive haemorrhage in fractures of the pelvis. Southern Medical Journal 1963;56:933-8.

2. Wilson JS, Miranda A, Johnson BL, et al. Vascular injuries associated with elective orthopaedic procedures. Ann Vasc Surg 2003;17:641-4.

3. Aksoy M, Taviloglu, Yanar H, et al. Percutaneous transcatheter embolisation in arterial injuries of the lower limbs. Acta Radiol 2005;46:471-5.

4. Sundaram M, Patel B, Wolverson MK, Riaz MA. Superior gluteal artery hemorrhage following pelvic fractures controlled by embolisation. Clin Radiol 1981;32:187-
90 . 